\title{
NEUROCOGNITIVE PROFILES OF PATIENTS WITH THE FIRST EPISODE OF PSYCHOSIS AND SCHIZOPHRENIA DO NOT DIFFER QUALITATIVELY: A NESTED CROSS-SECTIONAL STUDY
}

\author{
Dina Bosnjak Kuharic ${ }^{1^{*}}$, Porin Makaric ${ }^{1^{*}}$, Ivana Kekin², Zarko Bajic ${ }^{3}$, Maja Zivkovic ${ }^{2}$, \\ Aleksandar Savic ${ }^{1,4}$, Drazenka Ostojic ${ }^{1,5}$, Vlado Jukic $^{1,4}$ \& Martina Rojnic Kuzman ${ }^{2,4}$ \\ ${ }^{1}$ University Psychiatric Hospital Vrapce, Zagreb, Croatia \\ ${ }^{2}$ Department of Psychiatry, Zagreb University Hospital Centre, Zagreb, Croatia \\ ${ }^{3}$ Biometrika Healthcare Research, Zagreb, Croatia \\ ${ }^{4}$ Zagreb School of Medicine, University of Zagreb, Zagreb, Croatia \\ ${ }^{5}$ Faculty of Law, University of Zagreb, Zagreb, Croatia
}

"These authors have contributed equally to this manuscript

received: 31.5 .2018 ;

revised: 7.8.2018;

accepted: 20.11 .2018

\section{SUMMARY}

Background: The aim of study was to analyze neurocognitive profiles in patients with first-episode psychosis (FEP) and patients with schizophrenia (SCH), and their correlations with other clinical features.

Subjects and methods: We performed a multicentric cross sectional study including 100 FEP and 100 SCH recruited from three Croatian hospitals during 2015-2017. Assessment included a set of neurocognitive tests, psychiatric scales and self-reporting questionnaires. The main analysis was done by multigroup latent profile analysis.

Results: Multigroup latent profile analysis resulted in three structurally equivalent neurocognitive profiles ("Best", "Medium", "Worst"), with differences in the severity of neurocognitive deficits measured with successfulness in solving domain specific tasks. The "Best" profile was statistically significantly more prevalent in FEP and "Worst" profile in the SCH. Negative symptom score was the highest in patients with the "Worst" profile and the lowest among those with the "Best" profiles.

Conclusions: Differences in neurocognitive profiles between FEP and SCH appear to be quantitative rather than qualitative nature, possibly reflecting a specific trait of illness that may progress over time. Defining neurocognitive profiles from the first episode of psychosis could help in tailoring individualized treatment options with focus on neurocognitive and negative symptoms and possible influence on patients' overall clinical outcome.

Key words: neurocognitive impairment - neurocognitive profile - psychotic disorders - first-episode psychosis - schizophrenia

\section{INTRODUCTION}

Schizophrenia is a long-term, recurrent disorder characterized by episodes of acute psychosis and their remission (Clementz et al. 2015). While the clinical presentation may be very heterogeneous, generally, the illness usually starts with a prodromal period followed by the first episode of acute psychosis. It is characterized by symptoms that can be divided into five dimensions: positive, negative, neurocognitive, affective and psychomotor. During the following months most patients achieve remission of the first episode usually followed by reoccurring periods of acute psychosis and remission until a stage of residual phase is reached (Wiersma et al. 1998). In contrast to the first episode, every new episode usually requires more time and more medication to achieve remission (Andreasen et al. 2005).

Neurocognitive symptoms are often defined as the core feature of schizophrenia (Barder et al. 2013). Deficits in cognitive functioning are typically 0.5 to 2 standard deviations below healthy control group across a number of cognitive domains (Gold 2004, Keefe
2008). Cognitive impairment seems to be present even in the premorbid and prodromal phases of illness (Bora \& Murray 2014, Eisenacher et al. 2016, Fusar-Poli et al. 2012) with a significant decline in all cognitive domains in the first episode and early phase of illness (Mesholam-Gately et al. 2009).

From the clinical point of view, neurocognitive symptoms are relevant as they may be regarded as the most resistant to pharmacological treatment compared to all other symptom dimensions (Ahmed \& Bhat 2014, Trampush et al. 2015). Moreover, they seem to be connected with different aspects of functionality as an objective measure and quality of life as a subjective measure of illness outcome (Lepage et al. 2014, Mesholam-Gately et al. 2009, Rodriguez et al. 2015). Neurocognitive decline is directly associated with poor psychosocial functioning, low work productivity and high absenteeism of persons with schizophrenia and consequently present a significant emotional and economic burden to them and the society (Chong et al. 2016, Fett et al. 2011, Kane \& Lencz 2008, Rodriguez et al. 2015). 
Despite the clinical relevance of neurocognitive decline in schizophrenia, there is a number of uncertainties in the occurrence and progression of these symptoms in schizophrenia. First, it is rather unclear which are the factors influencing neurocognitive decline over time in patients with schizophrenia $(\mathrm{SCH})$. In the current literature, neurocognitive decline over time in $\mathrm{SCH}$ was found to be associated with male gender, unemployment, poor premorbid achievement, later age of onset, presence of negative symptoms (Bora 2015, Bora et al. 2009), suicidality (Ventriglio et al. 2016) and lower achievement of stable remission during the first year of illness (On et al. 2016, Rund et al. 2016).

Second, it is unclear whether there are differences in the speed and severity of neurocognitive decline among different neurocognitive functions. Although majority of longitudinal studies report relative stability of neurocognitive decline during the course of illness (Hoff et al. 2005, Rund et al. 2016), some report different changes in specific neurocognitive domains. According to Barder et al. (2013) working memory significantly improved during the first two years of follow-up of patients with first episode of psychosis (FEP), with no further changes in the following three years. However, other authors report decline in some of the domains, e.g. in visuospatial abilities (Stirling et al. 2003) or memory and speed of processing (Øie et al. 2008). Apart from a number of factors that can explain these results, the heterogeneity of assessment methods and applied neurocognitive tests may further contribute to it. Generally, different neurocognitive tests are used for the assessment of specific neurocognitive domains (Mesholam-Gately et al. 2009). In order to avoid multiplication, attempts were made to produce neurocognitive test batteries, for example with The Measurement and Treatment Research to Improve Cognition in Schizophrenia (MATRICS) Consensus Cognitive Battery (Nuechterlein \& Green 2006). However, the dimensional structure of these batteries is still not consistently reproduced, at least in the international, European cultural context (Mohn et al. 2017). Thus, the influence of different cultural context on results of psychological tests which are translated from one country to another is not defined.

To overcome these limitations, we conducted this study aiming to construct comprehensive neurocognitive profiles in schizophrenia, by applying a number of individual neurocognitive tests previously used in the Croatian population. Tests assessed five neurocognitive domains in two large different groups of patients with schizophrenia: the patients with the first episode and patients with multiple episodes. Secondly, we attempted to analyze the correlations of obtained profiles with clinical features of patients and their perceived quality of life.

\section{SUBJECTS AND METHODS}

\section{Study protocol}

This cross-sectional study was conducted at three Croatian hospitals: Zagreb University Hospital Centre, Neuropsychiatric Hospital Dr. Ivan Barbot, and University Psychiatric Hospital Vrapce. It was nested within the prospective cohort study: "Biomarkers in schizophrenia-integration of complementary methods in longitudinal follow up of FEP patients". The power analysis was done for the main prospective cohort study, and it was not conducted specifically for this analysis. Patients were enrolled between June 2015 and January 2017. The study protocol was approved by the Ethics Committees of the participating institutions, and all participants gave their informed consent before the inclusion. The study was conducted in accordance with World Medical Association Declaration of Helsinki 2013 (World Medical Association 2013).

\section{Participants}

Participants were recruited proportionally at the participating hospitals until the sample sizes reached 100 participants from each population. Inclusion criteria for FEP were: no history of antipsychotic use prior to admission to hospital, first episode of psychosis with the fulfillment of the criteria for psychotic episode (codes F23, F29) according to the criteria of International Classification of Disorders, $10^{\text {th }}$ revision (ICD-10) (World Health Organization 1992). Inclusion criteria for $\mathrm{SCH}$ were: $\geq 2$ acute psychotic episodes, illness duration $>5$ years and fulfillment of criteria for schizophrenia spectrum disorders (code F20) according to the ICD-10 criteria (World Health Organization 1992). Exclusion criteria for both groups were: $<18$ years of age, mental retardation, mental illness in childhood that can present with psychosis, neurological disorders, pregnancy and lactation, organic psychosis, the use of medications that can produce psychotic reactions, comorbid alcoholism or other addictions, use of drugs (included were patients who consummated marijuana no more than 3 times a year), psychiatric confinement and legally incapacitated participants. All patients were assessed during the acute phase of the illness at up to three weeks of treatment. The neurocognitive assessment took approximately 60 minutes and solving other questionnaires additional 20 minutes. None of the participants discontinued the assessments. Sociodemographic and clinical characteristics of FEP and $\mathrm{SCH}$ are presented in Table 1.

\section{Assessment of participants and expected outcomes}

Our primary outcome was the neurocognitive profile in patients with first episode psychosis and patients with schizophrenia. We used Mini Mental Status Examination 
Table 1. Patients sociodemographic and clinical characteristics; only cases with complete data on neurocognitive tests

\begin{tabular}{|c|c|c|}
\hline & $\begin{array}{c}\text { FEP } \\
(\mathrm{n}=87)\end{array}$ & $\begin{array}{c}\mathrm{SCH} \\
(\mathrm{n}=90)\end{array}$ \\
\hline \multicolumn{3}{|l|}{ Sociodemographic characteristics } \\
\hline \multicolumn{3}{|l|}{ Sex } \\
\hline male & $53(60.9)$ & $47(52.2)$ \\
\hline female & $34(39.1)$ & $43(47.8)$ \\
\hline Age (years), mean (SD) & $28(9.7)$ & $39(11.0)$ \\
\hline \multicolumn{3}{|l|}{ Education } \\
\hline primary & $8(9.2)$ & $13(14.4)$ \\
\hline secondary & $50(57.5)$ & $63(70.0)$ \\
\hline university or students & $29(33.3)$ & $14(15.6)$ \\
\hline Being in a relationship & $18(20.7)$ & $20(22.2)$ \\
\hline Being employed & $25(28.7)$ & $14(15.6)$ \\
\hline Positive family anamnesis & $37(42.5)$ & $39(43.3)$ \\
\hline \multicolumn{3}{|l|}{ Clinical characteristics } \\
\hline Onset of illness (years), mean (SD) & $27(9.8)$ & $28(9.7)$ \\
\hline \multicolumn{3}{|l|}{ Antipsychotics } \\
\hline monotherapy & $45(51.7)$ & $36(40.0)$ \\
\hline combination therapy & $42(48.3)$ & $54(60.0)$ \\
\hline \multicolumn{3}{|l|}{ Antipsychotics type* } \\
\hline $1^{\text {st }}$ generation & $31(36.1)$ & $32(35.0)$ \\
\hline $2^{\text {nd }}$ generation & $82(94.4)$ & $65(72.5)$ \\
\hline clozapine & $8(9.7)$ & $38(42.5)$ \\
\hline Antidepressants & $4(4.2)$ & $18(20.0)$ \\
\hline Anxiolytics & $59(68.1)$ & $61(67.5)$ \\
\hline Mood stabilizers & $8(9.7)$ & $38(42.5)$ \\
\hline
\end{tabular}

Abbreviations: FEP = patients with first-episode psychosis; $\mathrm{SCH}=$ patients with schizophrenia; $\mathrm{SD}=$ standard deviation;

* Sum exceeds $100 \%$ due to the combination therapies Data are presented as number (percentage) of participants if not stated otherwise.

(MMSE) as a screening test for overall cognitive impairment (Folstein et al. 1975). MMSE result was not used in the definition of latent profiles, but as the additional explanatory variable. Neurocognitive profiles were made of five neurocognitive domains assessed with a battery of neurocognitive tests representing specific domains, as described below.

\section{Verbal memory}

Verbal memory was assessed by three tests and their subtests: Rey Auditory Verbal Learning Test (RAVLT) (Schmidt 1996), Wechsler verbal paired associates (Wechsler 1945) and Digit span test (Lichtenberger \& Kaufman 2009). RAVLT included three subtests: RAVLT A (immediate verbal memory of 15 random words, 5 repeating), RAVLT B (immediate verbal memory of another set of 15 random words) and RAVLT A30' (delayed verbal memory from RAVLT A list after 30 minutes). We used two subtests of Wechsler verbal paired associates (immediate recall of 6 series of verbal paired associates, Wechsler 6 , and delayed recall after 30 minutes, Wechsler 30'). Subtests of Digit span test were Digit span $\mathrm{F}$ for forward repeating of series of numbers and Digit span B for backwards repeating of series of numbers.

\section{Executive functions: problem solving and mental flexibility}

Executive functions were assessed by Block design test (Block design) (Hutt 1932), Frontal assessment battery (FAB) (Dubois et al. 2000), Clock drawing test (CDT) (Freedman et al. 1994) and subtests of Stroop test (Golden 1976) (Stroop colours, STROOP 2 and Stroop word-colours, STROOP 3) and Trial Making Test (Tombaugh 2004) (Trial making test B, TMTB).

\section{Attention and speed of processing}

Attention and speed of processing was assessed by Digit symbol test (Digit symbol) (Lichtenberger \& Kaufman 2009), subtest of Stroop Test (Golden 1976) (Stroop words, STROOP 1) and Trail Making Test (Tombaugh 2004) (Trial making test A, TMTA).

\section{Visuospatial abilities and delayed visual recall}

Two subtests of Rey-Osterrieth Complex Figure Test (Fastenau et al. 1999) were used for assessment of visuospatial abilities (ROCF) and delayed visual recall after 30 minutes (ROCF 30').

\section{Language functions}

Language functions were assessed by two tests: Semantic (category) and Phonetic fluency test (Semantic, Phonetic) (Lichtenberger \& Kaufman 2009). Score was calculated from the number of words generated in each category (categories "animals", "vegetables" and "supermarket" for semantic, and letters "a" and "f" for phonetic fluency).

Secondary outcome were correlations of neurocognitive profile in both patient groups with psychopathology and quality of life. Assessments included: 1) clinical rating of symptoms: Positive and Negative Syndrome Scale, PANSS (Kay et al. 1987) and Calgary Depression Scale for Schizophrenia, CDSS (Addington et al. 1990); 2) selfreport questionnaires: Inventory of Depressive Symptomatology-Self Report, IDS-SR (Rush et al. 1996), Barrat Impulsiveness Scale-11, BIS-11 (Patton \& Stanford 1995), Aggression questionnaire, AQ (Buss \& Perry 1992), The Holmes-Rahe Stress Inventory, STRESS (Holmes \& Rahe 1967), Questionnaire on suicide ideation and behavior, SUICIDE (Marušič et al. 2007) and World Health Organization Quality of Life Assessment, WHOQOL-BREF (World Health Organization Group 1998).

\section{Statistical analysis}

We enrolled $100 \mathrm{FEP}$ and $100 \mathrm{SCH}$. Data were missing on at least one neurocognitive test in 13 FEP and $10 \mathrm{SCH}$. Although Little's MCAR test did indicated that data were missing completely at random $\left(\chi^{2}=146.5\right.$; $\mathrm{df}=157 ; \mathrm{p}=0.716$ ) we did a sensitivity analysis on multiply imputed data after the main analysis done on the cases with complete data only. 
Table 2. Fit statistics for different models

\begin{tabular}{|c|c|c|c|c|c|c|}
\hline & LL & $\mathrm{np}$ & $\mathrm{BIC}_{\mathrm{LL}}$ & $\mathrm{CAIC}_{\mathrm{LL}}$ & BLRT & Entropy $\mathrm{R}^{2}$ \\
\hline \multicolumn{7}{|l|}{$\operatorname{FEP}(n=87)$} \\
\hline 1 profile & -494 & 10 & 1033 & 1043 & - & 1.00 \\
\hline 2 profiles & -419 & 21 & 932 & 953 & $<0.001$ & 0.85 \\
\hline 3 profiles & -377 & 32 & 896 & 928 & $<0.001$ & 0.89 \\
\hline 4 profiles & -359 & 43 & 909 & 952 & 0.008 & 0.89 \\
\hline 5 profiles & -346 & 54 & 933 & 987 & 0.068 & 0.95 \\
\hline \multicolumn{7}{|l|}{$\mathrm{SCH}(\mathrm{n}=90)$} \\
\hline 1 profile & -616 & 10 & 1278 & 1288 & - & 1.00 \\
\hline 2 profiles & -535 & 21 & 1165 & 1186 & $<0.001$ & 0.85 \\
\hline 3 profiles & -504 & 32 & 1152 & 1184 & $<0.001$ & 0.90 \\
\hline 4 profiles & -485 & 43 & 1164 & 1207 & 0.010 & 0.83 \\
\hline 5 profiles & -475 & 54 & 1192 & 1246 & 0.196 & 0.86 \\
\hline \multicolumn{7}{|l|}{ Similarity between FEP and SCH } \\
\hline Complete heterogeneity & -939 & 39 & 2077 & 2126 & & \\
\hline Within profiles means equal across $\mathrm{FEP}$ and $\mathrm{SCH}$ & -948 & 29 & 2029 & 2068 & & \\
\hline Complete homogeneity & -946 & 32 & 2057 & 2089 & & \\
\hline
\end{tabular}

Abbreviations: $\mathrm{LL}=\log$-likelihood; $\mathrm{np}=$ number of free parameters; $\mathrm{BIC}=$ Bayesian information criterion based on loglikelihood; $\mathrm{CAIC}=$ Consistent Akaike's information criterion based on log-likelihood; BLRT $=$ bootstrap likelihood ratio test between the model in row and the model in the first row above the tested one

Prior to analysis the results of four cognitive tests measuring time (STROOP, 2, 3, TMT-A, and TMT-B) were reversed by multiplying them with -1 and adding the maximum original value, so that higher values in all tests represent better cognitive functioning. To avoid multicollinearity, local dependence, and to increase reliability of measurements we did a categorical, nonlinear principal component analysis of all tests within each neurocognitive domain. In every domain only one categorical principal component was extracted. We determined the number of components within each domain by parallel analysis (Watkins 2006). In comparison of FEP and $\mathrm{SCH}$ we did not perform a testing of statistical significance of the differences because the only purpose of this comparison was the detection of potential confounders of our main result. The main analysis was done by multigroup latent profile analysis of these principal components. In the first step, we analyzed configure equivalence, that is: whether the same number of latent profiles had the best fit to the data in samples from both of our targeted populations. We did this by fitted a one-class model to the data separately in both samples, then increasing the number of classes one by one and fitted each model until no further improvement could be achieved. In the second step, we analyzed the structural similarity, that is: whether the particular neurocognitive tests' results were independent of our targeted populations; whether the profiles were characterized by similar neurocognitive tests' results in both samples; whether the nature of neurocognitive profiles was similar in FEP and $\mathrm{SCH}$ samples. We did this by fitting model where the neurocognitive tests results' within-profile means were constrained to be equal across two samples. We evaluated different models fit to the data by lowest Bayesian information criterion based on log-likelihood (BIC), Consistent Akaike's information criterion based on log-likelihood (CAIC), significant bootstrap likelihood ratio test between the model with $\mathrm{k}$ and $\mathrm{k}-1$ profiles (BLRT), and entropy $\mathrm{R}^{2}$ (Finch 2015). In both FEP and $\mathrm{SCH}$ sample, the models with three latent profiles had the best fit to the data (Table 2). Therefore, the configured equivalence was confirmed. The model where the within-profile means were constrained to be equal across the two samples fitted the data better than the completely unconstrained or completely homogenous models. We accessed a local independence by the inspection of bivariate residuals. By the protocol, if $>5 \%$ of participants have missing data on any neurocognitive test, we planned to do a sensitivity analysis on the data set with multiply imputed data. Multiple imputation was done by fully conditional specification of the iterative Markov chain Monte Carlo method (Rubin 2004). Whether the data were missing completely at random was assessed by Little's MCAR test (Little 1988). The level of statistical significance was set at two-tailed $\mathrm{p}<0.05$ and we gave all confidence intervals at $95 \%$ level. Correction for multiple testing was done by sequential Holm-Bonferroni correction. Statistical data analysis was performed by Latent GOLD version 5.1. and NCSS 11 Statistical Software (2016) (NCSS, LLC. Kaysville, Utah, USA).

\section{RESULTS}

The structural equivalence of three latent profiles across FEP and SCH samples of patients was confirmed. The three profiles were quantitatively and not qualitatively different (Table 3, Figure 1, Figure 2). 
Table 3. Univariate description of three neurocognitive profiles

\begin{tabular}{lccc}
\hline & $\begin{array}{c}\text { Profile 1 } \\
\text { "Best" }\end{array}$ & $\begin{array}{c}\text { Profile 2 } \\
\text { "Medium" }\end{array}$ & $\begin{array}{c}\text { Profile 3 } \\
\text { "Worst" }\end{array}$ \\
\hline Diagnosis, $n$ (\%) & & & \\
FEP & $35(40.2)$ & $46(52.9)$ & $6(6.9)$ \\
SCH & $9(10.0)$ & $41(45.6)$ & $40(44.4)$ \\
Neurocognitive tests, mean (SD) & & & \\
Verbal memory & & & \\
RAVLT A & $47(8.7)$ & $34(9.2)$ & $22(8.8)$ \\
RAVLT B & $7(2.0)$ & $5(1.6)$ & $4(2.2)$ \\
RAVLT A 30 & $33(7.9)$ & $20(7.3)$ & $13(7.5)$ \\
Wechsler 6 & $43(6.1)$ & $38(8.1)$ & $30(11.4)$ \\
Wechsler 30 & $8(0.8)$ & $7(1.4)$ & $5(2.2)$ \\
Digit span F & $12(0.1)$ & $11(1.8)$ & $9(2.2)$ \\
Digit span B & $10(2.1)$ & $9(2.4)$ & $5(2.2)$ \\
Executive functions & & & \\
Block design & $62(3.6)$ & $47(14.5)$ & $33(15.2)$ \\
FAB & $18(0.4)$ & $17(1.4)$ & $14(3.2)$ \\
STROOP 2 & $364(23.3)$ & $328(46.1)$ & $269(72.1)$ \\
STROOP 3 & $849(32.3)$ & $791(67.1)$ & $687(183.3)$ \\
TMTB & $403(41.1)$ & $347(74.3)$ & $315(119.8)$ \\
CDT & $10(0.5)$ & $9(1.5)$ & $6(2.6)$ \\
Attention and speed of processing & & & \\
STROOP 1 & $221(17.7)$ & $194(34.6)$ & $152(53.0)$ \\
TMTA & $296(10.3)$ & $276(26.8)$ & $229(65.9)$ \\
Digit symbol & $46(13.2)$ & $37(12.2)$ & $21(8.5)$ \\
Visuospatial abilities & & & \\
ROCF & $35(2.5)$ & $35(1.7)$ & $26(9.8)$ \\
ROCF 30 & $20(7.1)$ & $13(7.4)$ & $5(5.0)$ \\
Language functions & & & \\
Semantic & $51(10.2)$ & $39(10.1)$ & $30(8.6)$ \\
Phonetic & $17(4.9)$ & $12(4.3)$ & $9(4.6)$ \\
Mini Mental Status Examination & $29(0.8)$ & $29(1.5)$ & $27(3.0)$ \\
\hline Abbrat & $5(3-0)$ & \\
\end{tabular}

Abbreviations: FEP = patients with first-episode psychosis; $\mathrm{SCH}=$ patients with schizophrenia; $\mathrm{SD}=$ standard deviation; RAVLT = Rey Auditory Verbal Learning Test; Wechsler $=$ Wechsler verbal paired associates; Digit span F and B = Digit span Forwards and Backwards; $\mathrm{FAB}=$ Frontal assessment battery; $\mathrm{TMTA}$ and $\mathrm{TMTB}=$ Trial making test A and $\mathrm{B}$; ROCF $=$ Rey-Osterrieth Complex Figure Test; Semantic $=$ Semantic (category) fluency test; Phonetic $=$ Phonetic fluency test

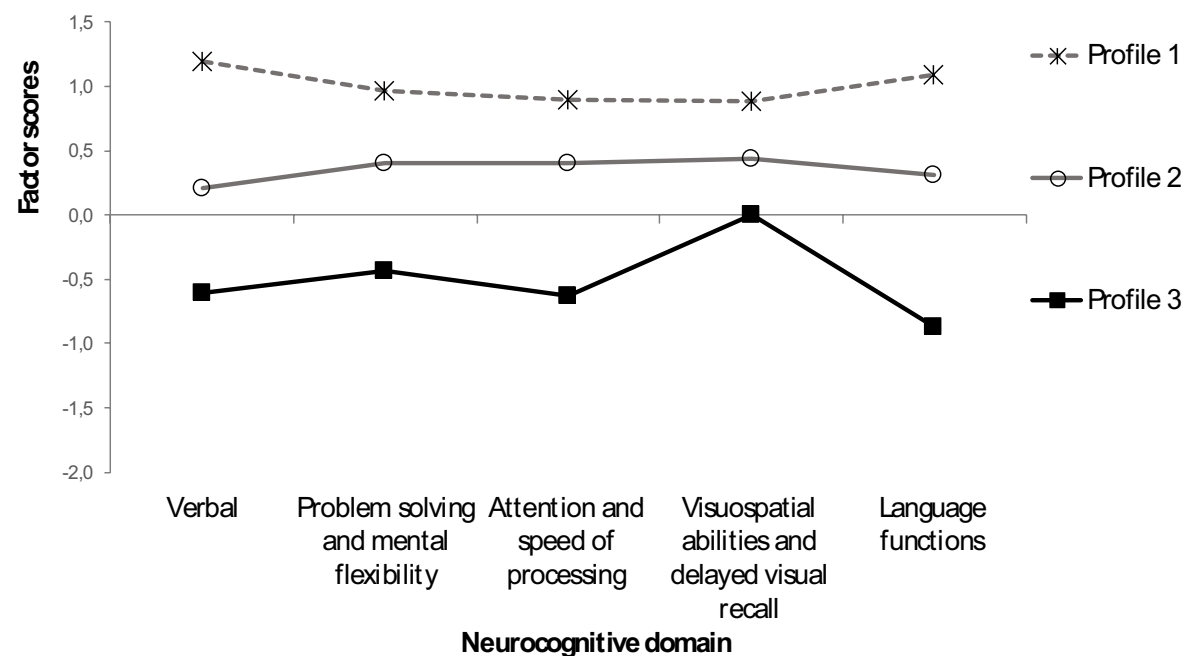

There were no statistically significant differences among the scores of the three neurocognitive profiles in FEP

Figure 1. Neurocognitive profiles in FEP $(\mathrm{n}=87)$ 


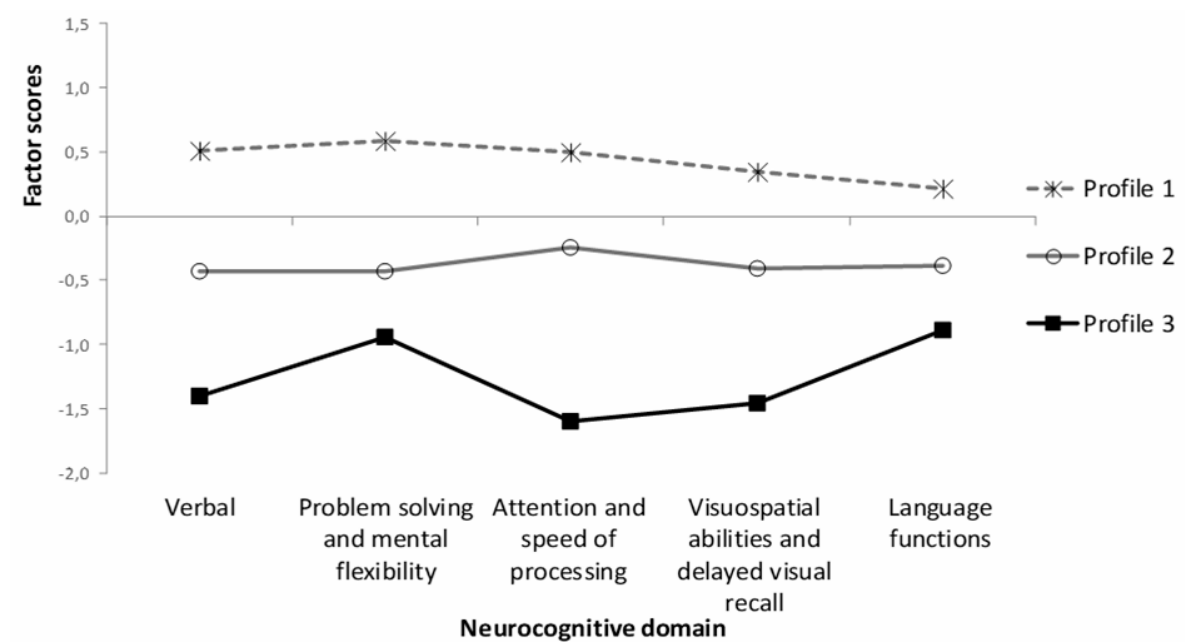

There were no statistically significant differences among the scores of the three neurocognitive profiles in SCH

Figure 2. Neurocognitive profiles in $\mathrm{SCH}(\mathrm{n}=90)$

Table 4. Differences in clinical characteristics between three neurocognitive profiles in FEP and SCH adjusted for age and education

\begin{tabular}{|c|c|c|c|c|c|c|c|c|c|}
\hline & $\begin{array}{l}\text { Profile } 1 \\
\text { "Best" } \\
(n=35)\end{array}$ & $\begin{array}{c}\text { FEP } \\
\text { Profile 2 } \\
\text { "Medium" } \\
(\mathrm{n}=46)\end{array}$ & $\begin{array}{c}\text { Profile } 3 \\
\text { "Worst" } \\
(n=6)\end{array}$ & $\begin{array}{l}\text { Profile 1 } \\
\text { "Best" } \\
(n=9)\end{array}$ & $\begin{array}{c}\mathrm{SCH} \\
\text { Profile 2 } \\
\text { "Medium" } \\
(\mathrm{n}=41)\end{array}$ & $\begin{array}{l}\text { Profile } 3 \\
\text { "Worst" } \\
(\mathrm{n}=40)\end{array}$ & $\begin{array}{c}\mathrm{p}_{1} \\
\text { diagnosis }\end{array}$ & $\begin{array}{c}\mathrm{p}_{2} \\
\text { profile }\end{array}$ & $\stackrel{\mathrm{p}_{3}}{\text { interaction }}$ \\
\hline PANSS total & $100(23.4)$ & $106(19.2)$ & $122(22.2)$ & $85(20.0)$ & $96(25.9)$ & $95(25.1)$ & 0.002 & 0.127 & 0.401 \\
\hline Positive symptoms & $26(10.3)$ & $28(6.5)$ & $31(4.7)$ & $22(6.6)$ & $23(10.0)$ & $26(9.6)$ & 0.023 & 0.504 & 0.985 \\
\hline Negative symptoms & $22(6.4)$ & $26(6.8)$ & $33(7.5)$ & $23(6.2)$ & $26(6.8)$ & $26(7.0)$ & 0.202 & 0.010 & 0.102 \\
\hline General symptoms & $51(11.0)$ & $52(9.3)$ & $59(12.2)$ & $40(9.1)$ & $46(12.2)$ & $43(11.3)$ & $<0.001$ & 0.290 & 0.184 \\
\hline \multicolumn{10}{|l|}{ Quality of life } \\
\hline WHOQOL-BREF total & $102(12.2)$ & $96(14.1)$ & $101(17.7)$ & $98(15.8)$ & $89(16.4)$ & $91(18.3)$ & 0.133 & 0.107 & 0.904 \\
\hline Physical health & 78 (11.7) & $71(16.2)$ & $76(18.6)$ & $76(17.9)$ & $61(20.0)$ & $62(22.2)$ & 0.165 & 0.056 & 0.549 \\
\hline Psychological health & $73(14.4)$ & $65(20.0)$ & $67(25.5)$ & $61(22.4)$ & $57(20.1)$ & $62(22.6)$ & 0.118 & 0.352 & 0.689 \\
\hline Social relationship & $68(16.6)$ & $58(22.0)$ & $64(33.4)$ & $56(13.1)$ & $55(23.6)$ & $52(27.1)$ & 0.066 & 0.539 & 0.624 \\
\hline Environment & $75(12.6)$ & $73(17.7)$ & $78(14.8)$ & $76(16.7)$ & $70(17.2)$ & $72(20.4)$ & 0.909 & 0.493 & 0.921 \\
\hline Suicide & $36(2.8)$ & $36(2.8)$ & $37(0.0)$ & $35(3.0)$ & $35(3.8)$ & $35(3.5)$ & 0.035 & 0.834 & 0.675 \\
\hline BIS total & $67(11.1)$ & $66(10.3)$ & $63(7.7)$ & $63(10.7)$ & $65(13.8)$ & $62(10.4)$ & 0.428 & 0.558 & 0.867 \\
\hline Attention & $11(1.9)$ & $11(2.2)$ & $10(1.0)$ & $10(2.8)$ & $11(3.6)$ & $10(3.3)$ & 0.472 & 0.763 & 0.722 \\
\hline Cognitive instability & $5.7(1.64)$ & $5.6(1.95)$ & $5.8(1.33)$ & $5.9(2.57)$ & $5.8(1.84)$ & $5.8(2.08)$ & 0.520 & 0.953 & 0.997 \\
\hline Motor & $14(3.9)$ & $13(2.7)$ & $12(2.9)$ & $13(4.0)$ & $13(4.5)$ & $13(4.5)$ & 0.857 & 0.540 & 0.520 \\
\hline Perseverance & $8(2.0)$ & $8(2.4)$ & $8(2.0)$ & $8(1.6)$ & $8(2.2)$ & $7(1.9)$ & 0.264 & 0.460 & 0.959 \\
\hline Self-control & $15(3.3)$ & $16(3.5)$ & $15(2.8)$ & $13(3.8)$ & $14(3.8)$ & $13(2.8)$ & 0.001 & 0.480 & 0.771 \\
\hline Cognitive complexity & $12(3.0)$ & $12(2.6)$ & $12(2.0)$ & $12(3.2)$ & $14(3.2)$ & $13(3.2)$ & & & \\
\hline \multicolumn{10}{|l|}{$2^{\text {nd }}$ order } \\
\hline Attentional & $17(2.9)$ & $17(3.2)$ & $16(1.7)$ & $16(4.0)$ & $17(5.0)$ & $16(4.8)$ & 0.848 & 0.937 & 0.865 \\
\hline Motor & $22(5.1)$ & $21(4.0)$ & $19(3.9)$ & $21(4.8)$ & $20(5.4)$ & $20(4.5)$ & 0.725 & 0.397 & 0.709 \\
\hline Non planning & $28(5.7)$ & $28(5.4)$ & $28(3.8)$ & $24(5.7)$ & $27(6.0)$ & $26(4.7)$ & 0.044 & 0.336 & 0.549 \\
\hline Aggression questionnaire & $70(18.4)$ & $76(23.0)$ & $64(12.7)$ & $69(15.1)$ & $74(16.3)$ & $76(23.3)$ & 0.475 & 0.335 & 0.339 \\
\hline Physical & $23(5.4)$ & $24(6.7)$ & $21(5.2)$ & $22(3.5)$ & $23(6.1)$ & $23(8.3)$ & 0.899 & 0.456 & 0.648 \\
\hline Verbal & $12(4.0)$ & $14(4.7)$ & $14(3.4)$ & $13(2.9)$ & $14(3.6)$ & $14(5.1)$ & 0.176 & 0.322 & 0.724 \\
\hline Anger & $19(5.5)$ & $19(6.3)$ & $16(4.5)$ & $17(4.4)$ & $18(4.7)$ & $18(5.8)$ & 0.795 & 0.341 & 0.506 \\
\hline Hostility & $16(6.4)$ & $19(8.1)$ & $14(4.5)$ & $16(8.2)$ & $18(6.5)$ & $19(7.5)$ & 0.301 & 0.278 & 0.191 \\
\hline IDS-SR & $17(11.1)$ & $19(14.7)$ & $12(12.9)$ & $15(9.2)$ & $24(14.5)$ & $25(16.8)$ & 0.150 & 0.155 & 0.222 \\
\hline
\end{tabular}

Data are presented as mean (standard deviation)

Abbreviations: $\mathrm{FEP}=$ patients with first episode psychosis; $\mathrm{SCH}=$ patients with schizophrenia; $\mathrm{p}=$ statistical significance of the difference between FEP and SCH adjusted for age; $p_{1}=$ statistical significance of the difference between FEP and SCH adjusted for age and education; $\mathrm{p}_{2}=$ statistical significance of the difference between neurocognitive profiles adjusted for age and education; $\mathrm{p}_{3}=$ statistical significance of the interaction of diagnosis and the neurocognitive profile, adjusted for age and education;

PANSS = Positive and Negative Syndrome Scale; WHOQOL-BREF = World Health Organization Quality of Life Assessment; Suicide $=$ Suicide ideation and behavior scale; BIS = Barrat Impulsiveness Scale; IDS-SR = Inventory of Depressive SymptomatologySelf Report 
According to mean neurocognitive domains' results we named them: "Best", "Medium", and "Worst". The best neurocognitive profile was significantly more prevalent in FEP, and the worst one in SCH sample. Prevalence of the medium profile was not significantly different between the two targeted populations. After adjustment for all monitored sociodemographic and clinical patients' characteristics by multinomial logistic regression, odds for the medium neurocognitive profile compared to the odds for the best one, were not significantly different between the two samples (FEP $\mathrm{OR}=0.29 ; 95 \%$ CI $0.07-1.21 ; \mathrm{p}=0.090$ ). However, FEP had significantly lower odds for having the worst neurocognitive profile compared to the odds of having the best one $(\mathrm{OR}=0.09 ; 95 \% \mathrm{CI} 0.01-0.59 ; \mathrm{p}=0.013)$.

We found statistically significant statistical difference in negative PANSS sub-scores between the three neurocognitive profiles adjusted for age and education, with the highest negative symptoms in patients with the "Worst" profile, and the lowest among those with the "Best" profiles, without differences between the FEP and SCH groups. Also, we have not found significant differences in scores of other PANSS sub-scales, quality of life, suicide ideation, impulsiveness, aggression nor depressive symptoms between the three profiles in any of the two samples (Table 4). There was a number of statistically significant differences in the scores of clinical tests between the FEP and $\mathrm{SCH}$ sample, as stated in Table 4.

\section{DISCUSSION}

When comparing FEP to $\mathrm{SCH}$, we found that three neurocognitive profile models fitted the data the best in both samples. Profile 1 ("Best"), Profile 2 ("Medium") and Profile 3 ("Worst") consisted of five neurocognitive domains (verbal memory, executive functions, attention and speed of processing, visuospatial abilities, language functions) represented with adequate neurocognitive tests. The three profiles were structurally equivalent across FEP and $\mathrm{SCH}$, with differences only in the values of specific neurocognitive tests. All profile scores reflecting results were significantly better in FEP compared to $\mathrm{SCH}$. Profile 3 was more frequent among SCH group and Profile 1 among FEP group. We have not found any statistically significant differences in the level of impairment among specific neurocognitive domains across the sample, but rather an overall decrease of neurocognitive abilities.

Our results may possibly indicate that the difference in neurocognitive profiles between FEP and $\mathrm{SCH}$ are quantitative rather than qualitative. In other words, the nature of neurocognitive deficit might not change further after the onset of psychosis, but it may reflect a certain trait of the illness that may be present in an attenuated form from the beginning of the illness. This is in line with neurodevelopmental theory of schizo- phrenia with the core neurocognitive symptoms as results of aberrations during the development of the brain starting even before the illness onset (Bora 2015, Bora \& Murray 2014). According to previous literature, mild neurocognitive deficits are present in the persons at risk for developing psychosis compared to healthy control groups (Fusar-Poli et al. 2012, Lencz et al. 2006), but in lower intensity compared to persons with developed, full-blown psychosis in both first episode or developed illness (Corigliano et al. 2014). Although some authors report less impairment in FEP when compared to $\mathrm{SCH}$, at least on some cognitive tests (Addington \& Addington 2002, Braw et al. 2007, Townsend \& Norman 2004), the majority of previous studies show that further development of neurocognitive deficits after the illness onset is relatively stable, with similar impairments in FEP and SCH (Corigliano et al. 2014, Mesholam-Gately et al. 2009). McCleery et al. (2014) report subtle differences when comparing these patient groups with healthy controls (e.g. better results in working memory and social cognition in FEP than in $\mathrm{SCH}$ ), but state that the overall distribution of impairment did not statistically defer between FEP and SCH group. Our results also reflect this stability with structurally identical neurocognitive profiles presented in both patient groups. It seems that after the illness onset, during the acute phase of first episode of psychosis, the full range of neurocognitive deficits is present in the same form as it is later during the course of schizophrenia.

As all neurocognitive domains show deficits in all three profiles and in both patient groups, our results may indicate a diffuse, rather than a specific localized impairment. This is partially in line with previous studies. Although majority of them agree that there is an overall neurocognitive deficit, part of the authors point out some more specific impairments. For example, some studies highlight that speed of processing is more impaired than other domains in both FEP and $\mathrm{SCH}$ (McCleery et al. 2014), or suggest that this domain underlies other neurocognitive deficits in schizophrenia as a limiting factor for overall cognitive performance (Dickinson et al. 2007, Ojeda et al. 2012). Besides speed of processing, Mesholam-Gately's et al. meta-analysis (2009) reports impairment in immediate verbal memory as a possible domain specific deficit in FEP, along with diffuse impairment. Henry's et al. meta-analysis suggests higher deficits of semantic store in schizophrenia (Henry \& Crawford 2005), while some other studies mention executive functions or working memory (Braw et al. 2007, Gonzalez-Ortega et al. 2013). These specific differences could be the result of heterogeneity of measures used for assessment in previous studies, with some tests possibly being more sensitive than others. Furthermore, patient samples differed across studies regarding e.g. duration of illness or treatment, phase of the illness (acute phase, remission) or recruitment type (outpatients, hospitalized patients etc.). 
Although we have not found statistically significant specific domain deficits, we did observe differences in the level of impairment among specific neurocognitive domains in patients with "Worst" profile, especially among SCH, with a higher level of impairment in attention, speed of processing, visuospatial abilities and delayed visual recall. This may possibly reflect specific subgroup of patients who are characterized by more severe symptoms from their first episode. Additionally, we found significant differences in negative symptom scores between the three neurocognitive profiles, with the "Worst" profile having the highest scores on negative PANSS in patients without significant differences between FEP and SCH samples. Consequently, more severe specific neurocognitive deficits along with higher levels of negative symptoms at the beginning of illness may contribute to poorer treatment response or overall outcome of treatment in the later period in these patients. This was previously reported in literature, e. g. Milev et al. (2005) reported significant correlations between poorer neurocognitive performance in different cognitive domains and the severity of negative symptoms and recent metaanalysis by Bora \& Murray (2014) reported association of the persistence of negative symptoms with lack of improvement in neurocognitive functions at follow-up. In accordance with this studies, FEP and $\mathrm{SCH}$ with "Worst" profile and higher negative symptoms may be especially vulnerable and may require more of differently tailored treatment approaches including specific cognitive training methods (cognitive remediation, metacognitive training, additional pharmacological agents) from the beginning of the treatment compared to $\mathrm{FEP}$ or $\mathrm{SCH}$ with "Best" neurocognitive profile.

Besides significant differences among profiles regarding negative symptoms, we found no such differences regarding other psychotic symptoms in neither of our two samples of patients. This is concordant with schizophrenia literature. Previous studies generally agree that there are no correlations between neurocognition and positive symptoms (de Gracia Dominguez et al. 2009, Dibben et al. 2009, Ventura et al. 2009), and only minority of them showed connection with other symptoms like disorganization (Nieuwenstein et al. 2001, Ventura et al. 2010). However, our results showed no statistically significant differences between the three profiles with self-measurement scales for quality of life, aggression, depression, suicidality and impulsiveness. This is somewhat inconsistent with some of the previous studies that found that neurocognitive deficits in various domains significantly correlated with perceived quality of life (Rodriguez et al. 2015), suicidality (Ventriglio et al. 2016), impulsivity or aggression (Reinharth et al. 2014). Possible explanation could be the difference in the study method, as we investigated the correlations of clinical features with the whole neurocognitive profile of single patient and not with specific neurocognitive tests and on a group level. Another explanation is in the use of self-measurement scales in our and other studies that could be biased due to different reasons, e.g. misunderstanding, exaggerating or due to social-desirability bias.

The main limitation of our study is cross-sectional design due to which we could only describe the difference between two samples assessed at the different points during the disease life-span. We have no proves that our current $\mathrm{SCH}$ sample will be representative for the neurocognitive profile of our FEP sample in 10 years, nor that our current FEP sample was representative for the neurocognitive profile of our $\mathrm{SCH}$ sample 10 years ago. Our sample may not be treated as representative for the entire Croatian population of FEP nor SCH, although there is no ground for the assumption that characteristics of our sample are systematically different in different regions of the country. Due to age differences between our FEP and $\mathrm{SCH}$ group, we adjusted the analysis for age, but we cannot exclude the effects of other factors not included in the study analysis, such as illness duration or medication on neurocognitive status of our patients. Another limitation is that a part of our assessments (for quality of life, suicidality, impulsiveness and aggression) are self-measurement questionnaires which results could be potentially biased at some level.

\section{CONCLUSIONS}

Presented in a profile manner, neurocognitive differences between FEP and SCH seem to be more of a quantitative than of qualitative nature, suggesting that neurocognitive symptoms are specific trait of illness, present since its beginning and progressing over time. There is a significant association of the three neurocognitive profiles with negative symptoms: patients with the "Best" profiles have the lowest negative symptoms, and those with the "Worst" profile have the highest negative symptom scores, regardless of the number of psychotic episodes (first-episode or multiepisode). There is no association between the three neurocognitive profiles with other psychotic symptoms and clinical features. Putting it into the clinical context, patients who present with the worst neurocognitive profile in the first episode may also have severe negative symptoms and possible be of particular risk for further neurocognitive decline. Thus, definition of neurocognitive profiles can help tailoring individualized treatment options. Special focus and treating with methods specifically designed for neurocognitive and negative symptoms (e.g. cognitive remediation) may possibly influence treatment outcome and warrant further investigation. 


\section{Acknowledgements:}

We thank the following colleagues from University Psychiatric Hospital Vrapce, Zagreb, Croatia, for providing us with patients needed for conducting this research: Ante Silic, MD, PhD, Darko Perusic, MD, PhD, Mirna Sisek-Sprem, MD, PhD, Patricia Jelacic, MD, Natasa Duran, MD, Mladen Haupert, MD. We thank colleague from Department of Neurology, Clinical Hospital Centre Zagreb, Croatia, Marina Boban, MD, PhD for help and consultations regarding neurocognitive assessments.

\section{FUNDING}

This research was supported by the grant of the Croatian Science Foundation No UIP-2014-09-1245 Biomarkers in schizophrenia-integration of complementary methods in longitudinal follow up of FEP patients. The funding source was not involved in the collection, analysis and interpretation of data, in the writing of the report nor in the decision to submit the article for publication.

Conflict of interest: None to declare.

\section{Contribution of individual authors:}

Dina Bosnjak Kuharic, Porin Makaric \& Martina Rojnic Kuzman designed the study.

Maja Zivkovic, Aleksandar Savic, Drazenka Ostojic, Vlado Jukic \& Martina Rojnic Kuzman took part in recruitment and selection of patients, made diagnosis and performed psychopathology rating scales.

Dina Bosnjak Kuharic, Porin Makaric \& Ivana Kekin performed neurocognitive assessment of patients, organized self-measurement questionnaires and managed all data.

Zarko Bajic undertook statistical analysis and with Dina Bosnjak Kuharic \& Martina Rojnic Kuzman interpreted the data and presented them in tables and figures.

Dina Bosnjak Kuharic \& Porin Makaric wrote the first draft of the manuscript.

All authors drafted the manuscript, took part in writing and approved its final version.

\section{References}

1. Addington D, Addington $J \&$ Schissel B. A depression rating scale for schizophrenics. Schizophr Res 1990; 3:247-51

2. Addington $J \&$ Addington $D$. Cognitive functioning in first-episode schizophrenia. Journal of Psychiatry and Neuroscience 2002; 27:188

3. Ahmed $A O$ \& Bhat IA. Psychopharmacological treatment of neurocognitive deficits in people with schizophrenia: a review of old and new targets. CNS Drugs 2014; 28:301-18

4. Andreasen NC, Carpenter Jr WT, Kane JM, Lasser RA, Marder SR \& Weinberger DR. Remission in schizophrenia: proposed criteria and rationale for consensus. American Journal of Psychiatry 2005; 162:441-9
5. Barder HE, Sundet K, Rund BR, Evensen J, Haahr U, Ten Velden Hegelstad $W$, et al. Neurocognitive development in first episode psychosis 5 years follow-up: associations between illness severity and cognitive course. Schizophr Res 2013; 149:63-9

6. Bora E. Neurodevelopmental origin of cognitive impairment in schizophrenia. Psychol Med 2015; 45:1-9

7. Bora E \& Murray RM. Meta-analysis of cognitive deficits in ultra-high risk to psychosis and first-episode psychosis: do the cognitive deficits progress over, or after, the onset of psychosis? Schizophr Bull 2014; 40:744-55

8. Bora E, Yucel $M \&$ Pantelis C. Cognitive functioning in schizophrenia, schizoaffective disorder and affective psychoses: meta-analytic study. The British Journal of Psychiatry 2009; 195:475-82

9. Braw Y, Bloch Y, Mendelovich S, Ratzoni G, Gal G, Harari $H$, et al. Cognition in young schizophrenia outpatients: comparison of first-episode with multiepisode patients. Schizophrenia Bulletin 2007; 34:544-54

10. Buss AH \& Perry M. The aggression questionnaire. J Pers Soc Psychol 1992; 63:452-9

11. Chong HY, Teoh SL, Wu DB-C, Kotirum S, Chiou C-F \& Chaiyakunapruk N. Global economic burden of schizophrenia: a systematic review. Neuropsychiatric disease and treatment 2016; 12:357

12. Clementz BA, Sweeney JA, Hamm JP, Ivleva EI, Ethridge $L E$, Pearlson GD, et al. Identification of distinct psychosis biotypes using brain-based biomarkers. American Journal of Psychiatry 2015; 173:373-84

13. Corigliano V, De Carolis A, Trovini G, Dehning J, Di Pietro S, Curto M, et al. Neurocognition in schizophrenia: from prodrome to multi-episode illness. Psychiatry Res 2014; 220:129-34

14. de Gracia Dominguez M, Viechtbauer W, Simons CJ, van Os $J$ \& Krabbendam L. Are psychotic psychopathology and neurocognition orthogonal? A systematic review of their associations. Psychological bulletin 2009; 135:157

15. Dibben $C$, Rice $C$, Laws $K$ \& McKenna P. Is executive impairment associated with schizophrenic syndromes? A meta-analysis. Psychological medicine 2009; 39:381-92

16. Dickinson D, Ramsey ME \& Gold JM. Overlooking the obvious: a meta-analytic comparison of digit symbol coding tasks and other cognitive measures in schizophrenia. Arch Gen Psychiatry 2007; 64:532-42

17. Dubois B, Slachevsky A, Litvan I \& Pillon B. The FAB: a Frontal Assessment Battery at bedside. Neurology 2000; 55:1621-6

18. Eisenacher $S$, Rausch $F$, Ainser F, Englisch $S$, Becker A, Mier D, et al. Early cognitive basic symptoms are accompanied by neurocognitive impairment in patients with an 'at-risk mental state' for psychosis. Early Interv Psychiatry 2016

19. Fastenau PS, Denburg NL \& Hufford BJ. Adult norms for the Rey-Osterrieth Complex Figure Test and for supplemental recognition and matching trials from the Extended Complex Figure Test. Clin Neuropsychol 1999; 13:30-47

20. Fett A-KJ, Viechtbauer $W$, Penn DL, van Os $J \&$ Krabbendam L. The relationship between neurocognition and social cognition with functional outcomes in schizophrenia: a meta-analysis. Neuroscience \& Biobehavioral Reviews 2011; 35:573-88 
21. Finch H. A Comparison of Statistics for Assessing Model Invariance in Latent Class Analysis. Open J Stat 2015; 5:191-210

22. Folstein MF, Folstein SE \& McHugh PR. "Mini-mental state". A practical method for grading the cognitive state of patients for the clinician. J Psychiatr Res 1975; 12:189-98

23. Freedman M, Leach L, Kaplan E, Winocur G, Shulman K \& Delis DC. Clock Drawing: A Neuropsychological Analysis. Oxford University Press, 1994

24. Fusar-Poli P, Deste G, Smieskova R, Barlati S, Yung AR, Howes $O$, et al. Cognitive functioning in prodromal psychosis: a meta-analysis. Archives of general psychiatry 2012; 69:562-71

25. Gold JM. Cognitive deficits as treatment targets in schizophrenia. Schizophrenia research 2004; 72:21-8

26. Golden CJ. Identification of brain disorders by the Stroop Color and Word Test. J Clin Psychol 1976; 32:654-8

27. Gonzalez-Ortega I, de Los Mozos V, Echeburua E, Mezo $M$, Besga A, Ruiz de Azua S, et al. Working memory as a predictor of negative symptoms and functional outcome in first episode psychosis. Psychiatry Res 2013; 206:8-16

28. Henry $J \&$ Crawford $J$. A meta-analytic review of verbal fluency deficits in schizophrenia relative to other neurocognitive deficits. Cognitive neuropsychiatry 2005, 10:1-33

29. Hoff AL, Svetina C, Shields G, Stewart J \& DeLisi LE. Ten year longitudinal study of neuropsychological functioning subsequent to a first episode of schizophrenia. Schizophr Res 2005; 78:27-34

30. Holmes $T H \&$ Rahe $R H$. The social readjustment rating scale. Journal of psychosomatic research 1967; 11:213-8

31. Hutt ML. The Kohs block-design tests. A revision for clinical practice. Journal of Applied Psychology 1932; 16:298

32. Kane JM \& Lencz T. Cognitive deficits in schizophrenia: short-term and long-term. World Psychiatry 2008; 7:2930

33. Kay SR, Fiszbein A \& Opler LA. The Positive and Negative Syndrome Scale (PANSS) for Schizophrenia. Schizophrenia Bulletin 1987; 13:261-76

34. Keefe RS. Should cognitive impairment be included in the diagnostic criteria for schizophrenia? World Psychiatry 2008; 7:22-8

35. Lencz T, Smith CW, McLaughlin D, Auther A, Nakayama $E$, Hovey $L$, et al. Generalized and specific neurocognitive deficits in prodromal schizophrenia. Biological psychiatry 2006; 59:863-71

36. Lepage M, Bodnar $M \&$ Bowie CR. Neurocognition: clinical and functional outcomes in schizophrenia. Can J Psychiatry 2014; 59:5-12

37. Lichtenberger EO \& Kaufman AS. Essentials of WAIS-IV assessment. John Wiley \& Sons, 2009

38. Little RJ. A test of missing completely at random for multivariate data with missing values. Journal of the American Statistical Association 1988; 83:1198-202

39. Marušič A, Roskar $S \&$ Roškar M. Questionnaire on suicide ideation and behaviour. In: Shrivastava A (editor): Suicide prevention in developing countries, 201e9. Gaskell, 2007

40. McCleery A, Ventura J, Kern RS, Subotnik KL, GretchenDoorly D, Green $M F$, et al. Cognitive functioning in firstepisode schizophrenia: MATRICS Consensus Cognitive
Battery (MCCB) Profile of Impairment. Schizophr Res 2014; 157:33-9

41. Mesholam-Gately RI, Giuliano AJ, Goff KP, Faraone SV \& Seidman LJ. Neurocognition in first-episode schizophrenia: a meta-analytic review. Neuropsychology 2009; 23:315-36

42. Milev P, Ho BC, Arndt $S \&$ Andreasen NC. Predictive values of neurocognition and negative symptoms on functional outcome in schizophrenia: a longitudinal firstepisode study with 7-year follow-up. Am J Psychiatry 2005; 162:495-506

43. Mohn C, Lystad JU, Ueland T, Falkum E \& Rund BR. Factor analyzing the Norwegian MATRICS Consensus Cognitive Battery. Psychiatry and clinical neurosciences 2017; 71:336-45

44. Nieuwenstein MR, Aleman A \& de Haan EH. Relationship between symptom dimensions and neurocognitive functioning in schizophrenia: a meta-analysis of WCST and CPT studies. Journal of psychiatric research 2001; $35: 119-25$

45. Nuechterlein KH \& Green MF. MATRICS Consensus Cognitive Battery Manual. MATRICS Assessment Inc, Los Angeles, 2006

46. Øie M, Sundet $K \&$ Rund BR. Neurocognitive decline in early-onset schizophrenia compared with ADHD and normal controls: evidence from a 13-year follow-up study. Schizophrenia Bulletin 2008; 36:557-65

47. Ojeda N, Pena J, Schretlen D, Sanchez P, Aretouli E, Elizagarate E, et al. Hierarchical structure of the cognitive processes in schizophrenia: the fundamental role of processing speed. Schizophrenia research 2012; 135:72-8

48. On ZX, Cotton S, Farhall J, Killackey E \& Allott K. Relationship between duration of untreated psychosis and neurocognition and social cognition in first-episode psychosis. Schizophrenia research 2016; 176:529-32

49. Patton JH \& Stanford MS. Factor structure of the Barratt impulsiveness scale. Journal of clinical psychology 1995; 51:768-74

50. Reinharth J, Reynolds G, Dill C \& Serper M. Cognitive predictors of violence in schizophrenia: a meta-analytic review. Schizophrenia Research: Cognition 2014; 1:101-11

51. Rodriguez M, Spaniel F, Konradova L, Sedlakova K, Dvorska K, Prajsova J, et al. Comparison of Visuospatial and Verbal Abilities in First Psychotic Episode of Schizophrenia Spectrum Disorder: Impact on Global Functioning and Quality of Life. Front Behav Neurosci $2015 ; 9: 322$

52. Rubin DB. Multiple imputation for nonresponse in surveys. John Wiley \& Sons, 2004

53. Rund BR, Barder HE, Evensen J, Haahr U, ten Velden Hegelstad W, Joa I, et al. Neurocognition and Duration of Psychosis: A 10-year Follow-up of First-Episode Patients. Schizophr Bull 2016; 42:87-95

54. Rush AJ, Gullion CM, Basco MR, Jarrett RB \& Trivedi $M H$. The Inventory of Depressive Symptomatology (IDS): psychometric properties. Psychol Med 1996; 26:477-86

55. Schmidt M. Rey auditory verbal learning test: A handbook. Western Psychological Services Los Angeles, 1996

56. Stirling J, White C, Lewis S, Hopkins R, Tantam D, Huddy $A$, et al. Neurocognitive function and outcome in first-episode schizophrenia: a 10-year follow-up of an epidemiological cohort. Schizophrenia research 2003; 65:75-86 
57. Tombaugh TN. Trail Making Test A and B: normative data stratified by age and education. Arch Clin Neuropsychol 2004; 19:203-14

58. Townsend LA \& Norman RM. Course of cognitive functioning in first episode schizophrenia spectrum disorders. Expert Review of Neurotherapeutics 2004; 4:61-8

59. Trampush JW, Lencz T, DeRosse P, John M, Gallego JA, Petrides $G$, et al. Relationship of Cognition to Clinical Response in First-Episode Schizophrenia Spectrum Disorders. Schizophr Bull 2015; 41:1237-47

60. Ventriglio A, Gentile A, Bonfitto I, Stella E, Mari M, Steardo L, et al. Suicide in the Early Stage of Schizophrenia. Front Psychiatry 2016; 7:116

61. Ventura J, Hellemann GS, Thames AD, Koellner $V$ \& Nuechterlein KH. Symptoms as mediators of the relationship between neurocognition and functional outcome in schizophrenia: a meta-analysis. Schizophrenia research 2009; 113:189-99

62. Ventura J, Thames AD, Wood RC, Guzik LH \& Hellemann $G S$. Disorganization and reality distortion in schizophrenia: a meta-analysis of the relationship between positive symptoms and neurocognitive deficits. Schizophr Res 2010; 121:1-14

63. Watkins MW. Determining Parallel Analysis Criteria. J Mod Appl Stat Methods 2006; 5:344-6

64. Wechsler D. Wechsler memory scale. Psychological Corporation, San Antonio, 1945

65. Wiersma D, Nienhuis FJ, Slooff CJ \& Giel R. Natural course of schizophrenic disorders: a 15-year followup of a Dutch incidence cohort. Schizophrenia Bulletin 1998; 24:75

66. World Health Organization. The ICD-10 classification of mental and behavioural disorders: clinical descriptions and diagnostic guidelines. World Health Organization, Geneva, 1992

67. World Health Organization Group. Development of the World Health Organization WHOQOL-BREF quality of life assessment. Psychological medicine. 1998; 28:551-8

68. World Medical Association. World Medical Association Declaration of Helsinki: ethical principles for medical research involving human subjects. JAMA 2013; 310:2191-4

\section{Correspondence:}

Ass. Professor Martina Rojnic Kuzman, MD, PhD

Department of Psychiatry, Zagreb University Hospital Centre

Kišsatićeva 12, HR-10 000 Zagreb, Croatia

E-mail:mrojnic@gmail.com 